\title{
IPOMOEA IMPERATI (VAHL) GRISEB. (CONVOLVULACEAE): NUEVO XENÓFITO INVASOR EN LA PROVINCIA DE CÁDIZ (SUR DE ESPAÑA)
}

\author{
Juan GARCÍA-DE-LOMAS ${ }^{1}$, Elías D. DANA²*, Íñigo SÁNCHEZ-GARCÍA, \\ Jesús SÁNCHEZ ${ }^{4}$, Javier HERNÁNDEZ ${ }^{5}$, Francisco VASALLO ${ }^{6}$ y Guillermo CEBALLOS ${ }^{7}$ \\ ${ }^{1}$ Grupo de Investigación Estructura y Dinámica de Ecosistemas Acuáticos, \\ Universidad de Cádiz (España). \\ ${ }^{2}$ Grupo de Investigación, Transferencia I+D en Recursos Naturales, Universidad de Almería (España). \\ ${ }^{3}$ Zoobotánico de Jerez, C/Madreselva s/n. 11408, Jerez de la Frontera (Cádiz). \\ ${ }^{4}$ Urb. Punta Carnero, c/Caracolas 129, 11207, Algeciras (Cádiz). \\ ${ }^{5} \mathrm{C} /$ García de Sola $191^{\circ} \mathrm{D}, 11008$, Cádiz. \\ ${ }^{6}$ Avda. de los Naranjos 3, 11510, Puerto Real (Cádiz). \\ ${ }^{7}$ Servicio de Geo y Biodiversidad. Consejería de Medio Ambiente y Ordenación del Territorio, \\ Av. Manuel Siurot 50, 41071, Sevilla. \\ *Autor para correspondencia:edana@ual.es
}

Recibido el 26 de febrero de 2015, aceptado para su publicación el 12 de marzo de 2015

RESUMEN. Ipomoea imperati (Vahl) Griseb. (Convolvulaceae): nuevo xenófito invasor en la provincia de Cádiz (Sur de España. Se documentan los primeros registros de Ipomoea imperati (Vahl) Griseb. (Convolvulaceae) en la provincia de Cádiz. Esta especie rizomatosa y rastrera es nativa de Norteamérica y se ha encontrado formando poblaciones naturalizadas incipientes (entre $300-750 \mathrm{~m}^{2}$ ) en playas de los municipios de Barbate y Algeciras. Ipomoea imperati coloniza en ambos casos dunas móviles con diferente grado de desarrollo. El origen de la introducción es incierto. La evaluación del riesgo sugiere un riesgo alto de invasión, pudiendo extenderse la invasión sobre hábitats de interés comunitario.

Palabras clave. Plantas invasoras, riesgo de invasión, litoral, dunas, detección precoz.

\begin{abstract}
Ipomoea imperati (Vahl) Griseb. (Convolvulaceae): a new plant invader in the Cádiz province (Southern Spain). The first records of Ipomoea imperati (Vahl) Griseb (Convolvulaceae) in the province of Cádiz are documented. This rhizomatous and creeping species is native to North America. I. imperati was found as incipient naturalized populations (between $300-750 \mathrm{~m} 2$ ) on beaches from Barbate and Algeciras municipalities. In both cases, I. imperati colonizes shifting (yellow) dunes with different levels of development. The origin of the introduction is uncertain. The risk assessment resulted in a high risk of invasion of habitats of Community interest.
\end{abstract}

Key words. Invasive plants, risk of invasion, coast, dunes, early detection.

Este trabajo ha sido parcialmente financiado por la Consejería de Medio Ambiente y Ordenación del Territorio (Junta de Andalucía) a través del Programa Andaluz para el Control de las Especies Exóticas Invasoras. 


\section{INTRODUCCIÓN}

Las invasiones por plantas constituyen un serio impacto para la conservación de los ecosistemas naturales. El litoral es uno de los ambientes más propensos a sufrir invasiones biológicas. La fuerte demanda de esta estrecha franja del territorio por numerosas actividades (turismo de sol y playa, puertos comerciales y deportivos, industrias, etc.) da lugar a una elevada presión de propágulos que llegan de manera intencionada o accidental. Esto, unido a la existencia de un clima atemperado por el mar, da lugar a una elevada densidad de especies invasoras (e.g., Sanz-Elorza et al. 2004; Casimiro-Soriguer et al. 2008; García-deLomas et al. 2011). La detección temprana de plantas invasoras y las actuaciones de respuesta rápida incrementan las posibilidades de éxito en la gestión por diferentes motivos: pequeñas superficies, menores esfuerzos y costes en la gestión, menor tamaño del banco de semillas, impactos incipientes sobre la comunidad nativa, mayor rapidez de recuperación tras la actuación, etc. (Rejmánek \& Pitcairn, 2002). Por ello, es de gran utilidad para la gestión alertar sobre la presencia de nuevas especies invasoras en el territorio y poner en marcha actuaciones de respuesta rápida cuando sea posible.

Ipomoea imperati (comúnmente conocida como "Bejuco" o "Campanilla") es una convolvulácea perenne, rizomatosa y estolonífera originaria de zonas templadas y tropicales de América Central y SE de América del Norte (Silvestre, 2012). Es exclusiva de arenales costeros, donde crece formando densos tapices (McDonald, 1994; Silvestre, 2012). Los tallos son glabros y pueden alcanzar los $10 \mathrm{~m}$ de longitud y 2-7 mm de diámetro. Presentan raíces adventicias en los nudos y entrenudos. Las hojas son simples, pecioladas, glabras y coriáceas y de aspecto brillante, generalmente ovadas, ovado-elongadas o elípticas, de 2,2-5,2 $\mathrm{cm}$ de largo y 1,6-3 cm de ancho, con ápice obtuso y emarginado. Las flores son solitarias, axilares, con pedúnculos rojizos y glabros, de 1,3-5,5 cm de largo y aproximadamente $1 \mathrm{~mm}$ de diámetro. La corola es blanca e infundibular de 4,5-5,5 cm de ancho, glabra, con el tubo blanquecino, de 2,5-3 cm de largo por 4-6 mm de ancho. Sépalos subiguales, imbricados, pajizos, de 1-1,5 cm de largo por 4-6 $\mathrm{mm}$ de ancho, los exteriores coriáceos, los interiores membranáceos, glabros, con márgenes enteros y ápice agudo (McDonald, 1994).

En la Península Ibérica existen citas en las Islas Baleares, Valencia, Murcia y Huelva (Bonafé, 1979; Rigual, 1972; Peris et al. 1984; Mateo \& Aguilella, 1986; Cobo et al. 2005; Silvestre, 2012). Fuera de la Península, entre los territorios más próximos, también está presente en la costa atlántica de Marruecos (Hammada, 2007; Pérez-Latorre, com. pers.), sureste de Sicilia (Turrisi, 2001), islas Azores y Cabo Verde (Hansen \& Sunding, 1993), la isla de Porto Santo en el archipiélago de Madeira (Da Silva, 2002), y otros puntos de la Cuenca Mediterránea, especialmente hacia Oriente Medio (Turrisi, 2001, 2007). Estudios genéticos recientes sugieren que la introducción de estas poblaciones en el Mediterráneo y Norte de África se produjo a raíz de una dispersión transoceánica relativamente antigua, seguida de dispersión secundaria hacia el Mediterráneo (Cennamo et al. 2013).

En este trabajo se aportan dos nuevas citas en Andalucía, siendo las primeras para la provincia de Cádiz.

Ipomoea imperati (Vahl) Griseb.

CÁDIZ: UTM 30S 241849; 4005265, Playa del Retín, Barbate. 15-5-2012.

Pies adultos formando un rodal pequeño y disperso de unos $300 \mathrm{~m}^{2}$. Hábitat: duna embrionaria-primaria, de escaso desarrollo, en zona de avenidas con lascas (fig. 1). Entre las especies acompañantes están Sporobolus 

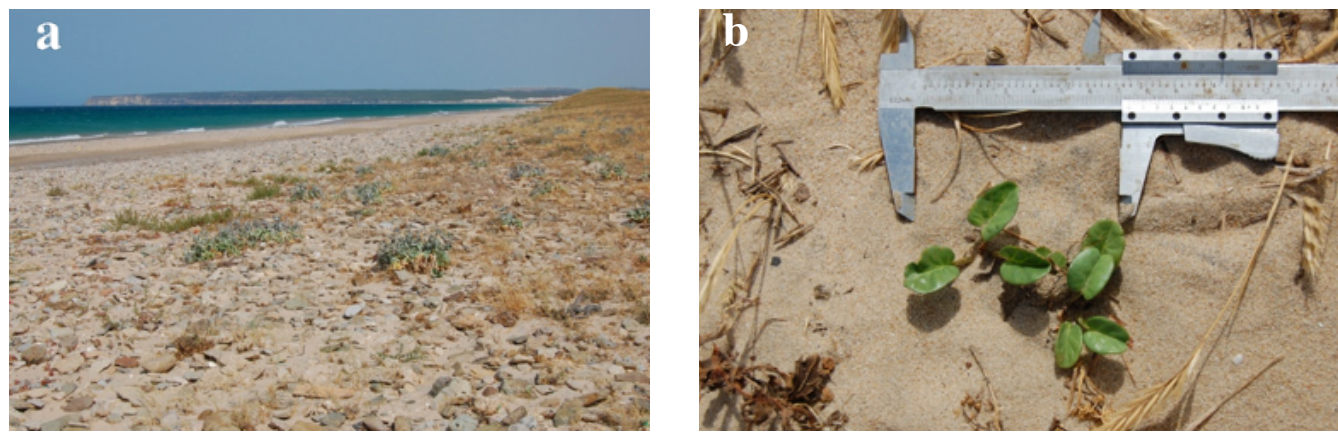

Figura 1. Tipo de hábitat colonizado por Ipomoea imperati en la playa del Retín (Barbate, Cádiz) (a) y detalle de un tallo joven (b). Habitat type colonized by Ipomoea imperati in El Retín beach (Barbate, Cádiz) (a) and detail of a young stem (b).
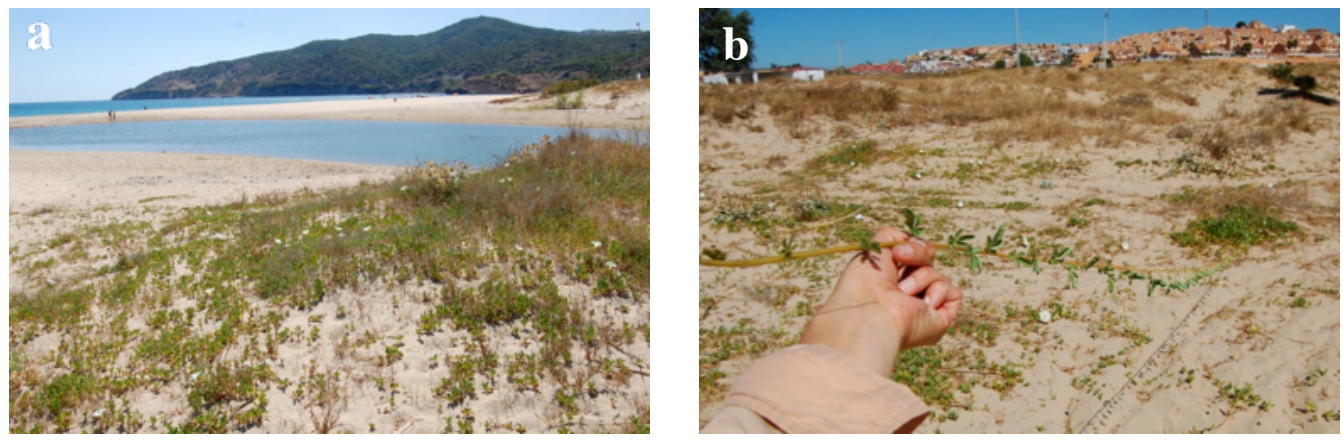

Figura 2. Tipo de hábitat colonizado por Ipomoea imperati en la playa de Getares (a) y detalle de uno de los largos tallos (b). Habitat type colonized by Ipomoea imperati in Getares beach (Algeciras, Cádiz) and a detail of a long stem (b).
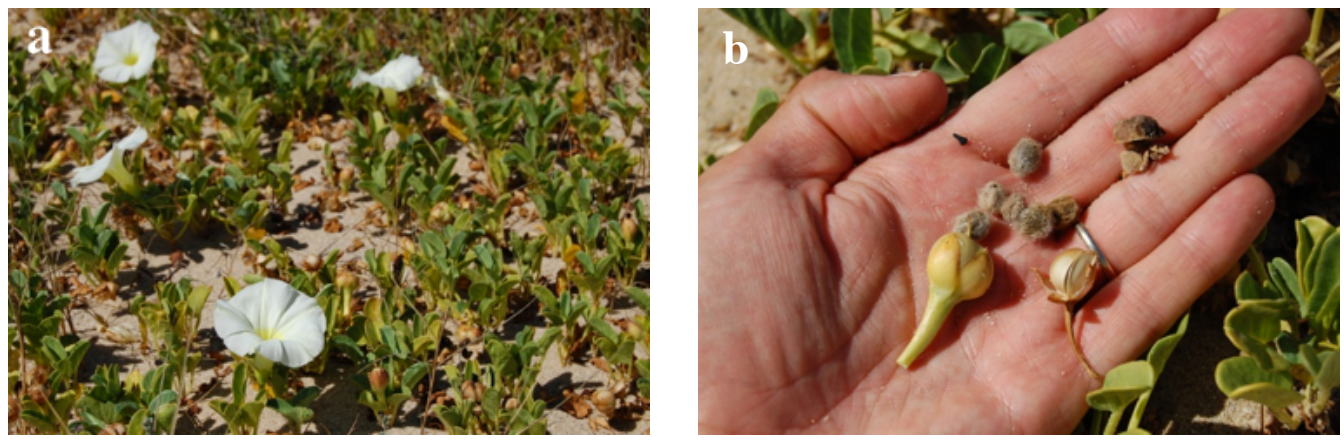

Figura 3. Ipomoea imperati en flor (a) y detalle de cápsulas y semillas (b) en la playa de Getares (Algeciras). Flowers (a), capsules and seeds (b) of Ipomoea imperati from Getares beach (Algeciras, Cádiz). 
pungens, Malcolmia littorea, Pancratium maritimum, Salsola kali, Hedypnois arenaria, Pseudorlaya pumila, Euphorbia peplis, Eryngium maritimum, Silene nicaeensis, Elymus farctus, Vulpia sp., Plantago coronopus, Medicago littoralis, etc. Origen incierto, aunque los ejercicios militares que se producen habitualmente en esta playa (Expósito, 2014) sugieren una introducción accidental, a través de materiales desembarcados de distinta procedencia. Estado: invasora. Xenófito holoagriófito.

CÁDIZ: UTM 30S 280017; 3997284, Playa de Getares, junto a la orilla izquierda de la desembocadura del río Pícaro, Algeciras. 4-9-2014. MGC 79869.

Pies adultos formando un rodal denso de unos $750 \mathrm{~m}^{2}$ (figs. 2, 3). En la fecha indicada la planta estaba en floración, con abundante presencia de cápsulas en diferentes grados de madurez y semillas (fig. 3b). Hábitat: Duna primaria. Entre las especies acompañantes están las propias de duna embrionaria y primaria, como Sporobolus pungens, Malcolmia littorea, Pancratium maritimum, Salsola kali, Hedypnois arenaria, Pseudorlaya pumila, Eryngium maritimum, Silene nicaeensis, Vulpia sp., Lotus creticus, Linaria pedunculata, Cakile maritima, etc. Desconocemos el origen de esta introducción. Estado: invasora. Xenófito hemiagriófito.

Como en otras ocasiones, el hallazgo se produce de manera casual, como consecuencia del aviso de un naturalista, lo que muestra la necesidad de generar redes sociales de alerta $\mathrm{y}$ equipos especializados en prospección del territorio e identificación y control de especies, cuya conjunción permite detectar taxones invasores en fases incipientes de asentamiento. Estas redes de alerta son especialmente útiles en casos como el que nos ocupa, en los que las poblaciones invasoras ocupan hábitats de alto valor para la conservación.

La novedad de las citas y el tamaño relativamente pequeño de los rodales sugieren una introducción reciente y un estado de invasión incipiente tanto en las nuevas localidades aportadas como en el conjunto de Andalucía. Por tal motivo, desde el Programa Andaluz para el Control de las Especies Exóticas Invasoras de la Consejería de Medio Ambiente y Ordenación del Territorio (CMAyOT), se realizó una evaluación del riesgo de invasión en base a la herramienta generada por la CMAyOT (García-de-Lomas et al. 2014), resultando un riesgo alto de invasión (anexo 1). Tiene especial relevancia el hecho de que Ipomoea imperati coloniza específicamente hábitats de interés comunitario como dunas móviles, recogidos en la Directiva 92/43/CE del Consejo, relativa a la conservación de los hábitats naturales y de la fauna y flora silvestres, y la Ley 42/2007, del Patrimonio Natural y la Biodiversidad.

Ipomoea imperati no está incluida en el Catálogo español de especies exóticas invasoras, regulado por el Real Decreto 630/2013. Esta especie parece haberse expandido sólo por vía natural o accidental, sin ser voluntariamente plantada o escapada de cultivos o jardines. Tampoco hay evidencias de ser objeto de cultivo en España (Sánchez de LorenzoCáceres, 2010). Sin embargo, la revisión de citas, la evaluación de riesgos y la constatación de su clara naturalización allí donde se ha introducido, recomienda la prohibición de su posible uso en el futuro.

\section{Anexo 1}

Resultado del análisis del riesgo de invasión de Ipomoea imperati con la herramienta de "Análisis del riesgo de invasión de vegetales exóticos (García-de-Lomas et al. 2014)

1) ¿La especie es hospedadora de plagas, parásita o vector de parásitos y patógenos para las especies silvestres autóctonas?: No

2) ¿Puede hibridarse con alguna especie autóctona?: No, el género de la especie exótica no está presente en la flora/fauna nativa 
Fuentes de información 1: Valdés et al. 1987.

Fuentes de información 2: Silvestre, 2012.

3) ¿Hay citas bibliográficas que reporten a esta especie como invasora, naturalizada o casual?: Las citas sugieren que la especie está naturalizada, casual o espontánea en la región de estudio

Fuentes de información 1: Cobo et al. 2005.

Fuentes de información 2: Cobo \& Bañuls, 2005.

4) ¿Es una especie potencialmente invasora según modelos de nicho ecológico y cambio climático aplicables a la región de estudio? (la resolución espacial del modelo debe ser al menos de $1 \mathrm{~km}$ ): No se sabe

5) ¿Es alelopática?: No se sabe

6) Indicar a qué tipo funcional pertenece la especie: Acuáticas, terófitos (plantas anuales y bianuales), geófito (p.ej. bulbosas, cañas) o hemicriptófitos cespitosos (p.ej. gramíneas perennes).

Fuentes de información 1: Lonard \& Judd, 1999

7) ¿Qué tipo de reproducción tiene?: Sexual (semillas viables)/esporas y vegetativa (bulbos, fragmentos de la planta, rebrote de cepa o raíz)

Fuentes de información 1: Lonard \& Judd, 1999

Fuentes de información 2: Silvestre, 2012.

8) ¿Qué periodo necesita para florecer/fructificar/ esporular/reproducirse por primera vez?: No se sabe

9) ¿Qué tamaño tienen las semillas/esporas/formas reproductivas vegetativas? En el caso de frutos indehiscentes, indicar el tamaño del fruto o "unidad dispersable": 0,5-1 cm

10) ¿Los propágulos (semillas o formas reproductivas vegetativas) durante cuánto tiempo son viables?: No se sabe

11) ¿Tiene dispersión zoócora, anemócora o hidrócora?: $\mathrm{Si}^{\prime}$

Fuentes de información 1: McDonald, 1994.

12) ¿Tiene otros mecanismos de dispersión no intencionada mediados por actividades humanas (vehiculos, contaminación de áridos, elevado tránsito de personas, etc.)?: No se sabe

13) ¿Es una planta fijadora de nitrógeno?: No

14) ¿Es capaz de reducir la llegada de luz al suelo o al bentos de más allá de lo propio del ambiente invadido, porque forme densos tapetes rastreros, acumule gran cantidad de hojas caidas o produzca gran cantidad de sombra?: $S i$

Fuentes de información 1: Lonard \& Judd, 1999.

Fuentes de información 2: McDonald, 1994
15) ¿Altera la frecuencia natural de perturbaciones (p.ej., fija arenas móviles, favorece incendios, modifica caudales, estrecha cauces de ríos...) en las zonas invadidas?: $S i$

Fuentes de información 1: Lonard \& Judd, 1999.

16) ¿Tiene tendencia a convertirse en la especie dominante o formar poblaciones monoespecíficas?: $S i$

Fuentes de información 1: Lonard \& Judd, 1999

17) ¿Coloniza, además de medios antropizados o alterados, medios en buen estado de conservación?: Sí. Los hábitats propicios están protegidos, constituyen hábitats de interés comunitario o albergan especies amenazadas

Fuentes de información 1: McDonald, 1999

Fuentes de información 2: Cobo et al. 2005

18) ¿La especie puede provocar problemas de salud pública?: No

19) ¿La especie puede provocar daños socioeconómicos?: No

AGRADECIMIENTOS. Los autores agradecen al Ministerio de Defensa los permisos para acceder a la zona y realizar las labores de control y seguimiento. A Borja Rodríguez, Salvador Domínguez, Vanesa Gámez y David M. García por su apoyo en los trabajos de eliminación. A los responsables del herbario MGC por las facilidades prestadas para el registro. Al Dr. Andrés Pérez Latorre y a Enrique Sánchez-Gullón las ideas aportadas sobre el germen de este trabajo.

\section{REFERENCIAS}

BONAFÉ, F. -1979- Flora de Mallorca vol. 3: 346. Editorial Moll, Mallorca. 1600 pp.

C A SIMIRO-SORIGUER, F. \& A.V. PÉREZ-LATORRE -2008- Aproximación al conocimiento de la flora alóctona de la provincia de Málaga (España): catálogo de metáfitos. Acta Botanica Malacitana 33: 1-10.

CENNAMO, P., E. DEL GUACCHIO, L. PAINO, O. DE CASTRO, B. MENALE, M. VÁZQUEZ-TORRES \& P. CAPUTO -2013- Genetic structure of Ipomoea imperati (Convolvulaceae) in the Mediterranean región and implications for its conservation. Phytotaxa 141: 40-54. 
COBO, M.D. \& S. BAÑULS -2005- Control de especies exóticas en el Parque Nacional de Doñana. Memoria de Resultados Año 2004. Ministerio de Medio Ambiente. http:// www.juntadeandalucia.es/medioambiente/ web/Bloques_Tematicos/Estado_Y_Calidad De_Los_Recursos_Naturales/Biodiversidad/ Redes_apoyo_gestion/especies_invasoras/ InformeFloraExotica2004.pdf. Acceso el 20 de febrero 2015.

COBO, M.D., E. SÁNCHEZ-GULLÓN \& P. WEICKERT -2005- Notas sobre la flora de Doñana. 4. Ipomoea imperati (Vahl) Griseb. (Convolvulaceae) nueva especie adventicia para el Parque Nacional de Doñana (SW España). Lagascalia 25: 248-249.

DA SILVA, R.M. -2002- Uma nova planta para o Archipélago da Madeira. Bocagiana 207: 1-7.

EXPÓSITO, J.L. -2014- En alerta máxima para la OTAN. Revista Española de Defensa 312: 28-33.

GARCÍA-DE-LOMAS, J., E. D. DANA, G. CEBALLOS \& F. ORTEGA -2014- Análisis del riesgo de invasión de vegetales exóticos. Manual práctico. Consejería de Medio Ambiente y Ordenación del Territorio, Junta de Andalucía. $50 \mathrm{pp}$.

GARCÍA-DE-LOMAS, J., I. SÁNCHEZ-GARCÍA, A. CÓZAR, E.D. DANA \& A.J. DELGADO -2011- Nuevas aportaciones a la xenoflora de la provincia de Cádiz. Lagascalia 31: 220-229.

HAMMADA, S. -2007- Etudes sur la vegetation des zones humides du Maroc. Catalogue et analyse de la biodiversité floristique et identification des principaux groupements végétaux. Thèse de Doctorat. Faculté des Sciences. Université Mohammed V-Agdal.

HANSEN, A. \& P. SUNDING -1993- Flora of Macaronesia. Checklist of vascular plants. 4 revised edit., Sommerfeltia 17: 1-295.

LONARD, R.I. \& F.W. JUDD -1999- The biological flora of coastal dunes and wetlands. Ipomoea imperati (Vahl) Griseb. Journal of Coastal Research 15: 645-652.

MATEO, G. \& A. AGUILELLA -1986- Notas florísticas Valencianas. V Folia Botanica Mis
5: 3-8.

MCDONALD, A. -1994- Flora de Veracruz. Convolvulaceae II. Instituto de Ecología, A.C., Xalapa, Veracruz, México. 66 pp.

PERIS, J.B., G. STÜBING \& E. GONZÁLEZABELLÁN -1984- Ipomoea stolonifera, un neófito litoral-psamófilo nuevo para la flora ibérica. Anales del Jardín Botánico de Madrid 40: 467-468.

REJMÁNEK, M. \& M.J. PITCAIRN -2002- When is eradication of exotic pest plants a realistic goal? En: Veitch, C.R. \& M.N. Clout (eds) Turning the tide: the eradication of invasive species. IUCN SSC Invasive Species Specialist Group. IUCN, Gland, Switzerland and Cambridge, UK, pp. 249-253.

RIGUAL, A. -1972- Flora y vegetación de la provincia de Alicante. Diputación Provincial de Alicante. Reeditada y ampliada en 1984. $403 \mathrm{pp}$.

SÁNCHEZ DE LORENZO-CÁCERES, J.M. -2010Convolvulaceae. En: Sánchez de LorenzoCáceres, J.M. (coord.) Flora ornamental española (Araliaceae $\cdot$ Boraginaceae), tomo VI. Consejería de Agricultura y Pesca, Junta de Andalucía, pp. 380-399.

SANZ-ELORZA, M, E.D. DANA \& E. SOBRINO -2004- Atlas de las plantas alóctonas invasoras en España. Dirección General para la Biodiversidad. Madrid, 384 pp.

SILVESTRE, S. -2012- Ipomoea L. En: S. Castroviejo et al. (Coord.). Flora Iberica 11: 279-286. C.S.I.C., Madrid.

TURRISI, R.E. -2001- Ipomoea imperati (Vahl) Griseb. (Convolvulaceae), nouvelle entité pour la flore sicilienne. Flora Mediterranea 11:373-378.

TURRISI, R.E. -2007-Importanza della conservazione in situ delle popolazioni di Ipomoea imperati (Vahl) Grisebach (Convolvulaceae) della Sicilia. Bollettino dell'Accademia Gioenia di Scienze Naturali 40: 141-148.

VALDÉS, B., S. TALAVERA \& E. FERNÁNDEZGALIANO -1987- Flora vascular de Andalucía Occidental, Vol. 2. Ed. Ketres, Barcelona, pp. 363-371. 